\title{
Interface states in junctions of two semiconductors with intersecting dispersion curves
}

\author{
A. V. Kolesnikov*, R. Lipperheide ${ }^{\dagger}$, A. P. Silin ${ }^{\ddagger}$ and U. Wille ${ }^{\dagger}$ \\ * Ruhr-University Bochum, Universitätstr. 150, D-447801, Bochum, Germany \\ $\dagger$ Bereich Theoretische Physik, Hahn-Meitner-Institut Berlin, \\ D-14091 Berlin, Germany \\ $\ddagger$ Tamm Theoretical Department of the Lebedev Physical Institute, RAS, \\ Leninskii pr. 53, 117924, Moscow, Russia
}

\begin{abstract}
PACS. 73.20.-r - Surface and interface electron states.
PACS. 73.20.Fz - Weak localization effects (e.g., quantized states).

PACS. 73.40.Lq - Other semiconductor-to-semiconductor contacts, p-n junctions, and heterojunctions.
\end{abstract}

\begin{abstract}
A novel type of shallow interface state in junctions of two semiconductors without band inversion is identified within the envelope function approximation, using the two-band model. It occurs in abrupt junctions when the interband velocity matrix elements of the two semiconductors differ and the bulk dispersion curves intersect. The in-plane dispersion of the interface state is found to be confined to a finite range of momenta centered around the point of intersection. These states turn out to exist also in graded junctions, with essentially the same properties as in the abrupt case.
\end{abstract}


The rapid progress in the synthesis of mesoscopic structures during the last decades has evoked renewed attention to the classic problems and methods of quantum mechanics. Precise thin-film deposition techniques allow of immediate tests of the theory, as for example, the experimental observation of localized "surface states" in AlGaAs/GaAs superlattices with a terminating layer of AlAs [1]. Such states were predicted by Tamm in 1932 [2]. These, as well as interface states (IS) in general, have since been under permanent investigation [3, [4, 5]. When the IS can be associated with the periodic potential of the lattice ("Shockley states") [6], they do not depend crucially on the details of the surface. Therefore, these IS, like the emulated IS of Ref. [1], can be studied within the envelope-function approach (EFA).

In applying the EFA to abrupt junctions, the matching conditions (MC) connecting the wave functions across the interface have to be established. It is known that in a heterojunction with band inversion, IS occur as an intrinsic property of the contact regardless of the precise type of MC [3, [4. In the more usual case of a heterojunction of two semiconductors with the same band symmetry, the role of the MC is more critical. The usual continuity conditions do not give rise to localized states, as is intuitively obvious when applying conventional quantum mechanics to a band-edge profile as shown in the inset in Fig. 1a. However, it was found for a model superlattice [5] that IS do occur, provided the wave functions are taken to be discontinuous at the interface. Unfortunately, within the EFA, the commonly used single-band description does not allow one to determine the MC unambiguously from the kinetic energy because the latter is not unique [7, 8].

In the present Letter, we infer unique $\mathrm{MC}$ for an arbitrary abrupt semiconductor junction within the EFA, using the two-band model [9]. A novel type of shallow IS is shown to occur in a contact of two semiconductors with bandgaps and effective masses such that their bulk dispersion curves intersect. This means that the interband velocity matrix element must vary across the junction so as to have a different 
bulk value on either side of the interface. It is found that interface states of this type are also present in graded heterojunctions.

In the two-band model, the four envelope functions combined in the spinor $\Psi$ satisfy an effective Dirac equation of the form $(\hbar=1)$

$$
\left(v \gamma^{0} \gamma \boldsymbol{p}+\gamma^{0} \Delta+\varphi\right) \Psi=\epsilon \Psi
$$

Here, $\gamma^{i} \equiv\left(\gamma^{0}, \gamma\right)$ are the Dirac matrices, $\Delta$ is half the energy gap, $\varphi$ is the work function, and $v$ is the interband velocity matrix element. With constant $\Delta, \varphi$, and $v$, one obtains the dispersion of a bulk semiconductor, $\epsilon^{( \pm)}(\mathbf{k})=\varphi \pm\left(\Delta^{2}+v^{2}|\mathbf{k}|^{2}\right)^{1 / 2}$, for the energy of an electron, $\epsilon^{(+)}$, and a hole, $\epsilon^{(-)}$, respectively. For $k v \ll \Delta$, electrons and holes have one and the same effective mass $m^{*}=\Delta / v^{2}$, given in terms of the parameters $\Delta$ and $v$ of the two-band model.

We now consider the case where $\Delta, \varphi$, and $v$ depend only on the z-coordinate in the direction of growth. Here, the momentum of free motion in the $(x, y)$-plane, $\mathbf{k}_{\perp}$, is conserved. The "pseudoparity" operator $P=i \gamma^{0} \gamma^{3}\left(\boldsymbol{\gamma}_{\perp} \mathbf{k}_{\perp}\right) / k_{\perp}$, which is the analogue of the helicity operator in Dirac theory, commutes with the Hamiltonian in (1) and has eigenvalues $\lambda= \pm 1$ [10]. Following Ref. [11], we write (1) for each $\lambda$ in the form of an eigenvalue problem for two scalar wave functions $\psi_{\lambda}$ and $\chi_{\lambda}$ of the two hybridized bands,

$$
\left(\begin{array}{cc}
\Delta+\varphi-\epsilon_{\lambda} & v \lambda k_{\perp}-v \partial_{z} \\
v \lambda k_{\perp}+v \partial_{z} & -\Delta+\varphi-\epsilon_{\lambda}
\end{array}\right)\left(\begin{array}{c}
\psi_{\lambda} \\
\chi_{\lambda}
\end{array}\right)=0
$$

For a graded system with a nonconstant $v$, the kinetic term $v \partial_{z}$ in (2) must be made Hermitian by replacing it with its symmetrized form $v(z) \partial_{z}+v^{\prime}(z) / 2$.

In the limit of an abrupt heterojunction with discontinuous $v(z)$, the term $v^{\prime}(z) / 2$ gives a $\delta$-function contribution in (1) and (2). For a consistent description, we introduce functions $f_{\lambda}$ and $g_{\lambda}$ by writing $\psi_{\lambda}=f_{\lambda} / \sqrt{v}$ and $\chi_{\lambda}=g_{\lambda} / \sqrt{v}$, so that $\left[v(z) \partial_{z}+v^{\prime}(z) / 2\right] f_{\lambda} / \sqrt{v}=\sqrt{v} f_{\lambda}^{\prime}$, and similarly for $g_{\lambda}$. It emerges that the two-band equation for $f_{\lambda}, g_{\lambda}$ with $z$-dependent $v$ has again the form (2), but the new functions $f_{\lambda}, g_{\lambda}$ are continuous at the interface $z=0$. The MC for the physical envelope 
function, satisfying the Hermitian version of (2), thus become discontinuous,

$$
\left.\psi_{\lambda} \sqrt{v}\right|_{-0}=\left.\psi_{\lambda} \sqrt{v}\right|_{+0},\left.\quad \chi_{\lambda} \sqrt{v}\right|_{-0}=\left.\chi_{\lambda} \sqrt{v}\right|_{+0}
$$

This type of MC departs from the widely used MC of a continuous envelope function across the interface [12, 13], established for heterojunctions with identical Bloch functions on either side, for which $v$ is constant. The assumption of constant $v^{2}=\Delta / m^{*}$ is rather well justified for most narrow-band heterojunctions of IIIV semiconductors. However, for most IV-VI and some III-V heterojunctions, $v$ does change appreciably across the interface [14], and the MC (3) should be used. A similar situation is discussed in Ref. [5] for a one-dimensional $\left(k_{\perp}=0\right)$ superlattice. In contrast to that work we consider the full three-dimensional case and derive the in-plane dispersion curves for the IS.

We study an abrupt contact of two homogeneous semiconductors with the parameters $\Delta_{i}, v_{i}$, and $\varphi_{i}\left(i=1,2\right.$ for $z<0, z>0$, respectively), where $\varphi_{1}=0, \varphi_{2}=-V$ (cf. the inset in Fig. 1a). The IS wave function has the form $\exp \left(+\kappa_{1} z\right), \exp \left(-\kappa_{2} z\right)$ for $z<0, z>0$, respectively, where $\kappa_{i}=\kappa_{i}\left(\epsilon, k_{\perp}\right)=+\left[k_{\perp}^{2}+\left(\Delta_{i}^{2}-\left(\epsilon-\varphi_{i}\right)^{2}\right) / v_{i}^{2}\right]^{1 / 2}$ $(i=1,2)$. The in-plane $\left(k_{z}=0\right)$ bulk dispersions $\epsilon_{i}^{( \pm)}\left(k_{\perp}\right)$ are found by setting $\kappa_{i}=0$

The bound-state eigenvalues are obtained from (2) with the aid of the MC (3), which leads to the eigenvalue equation

$$
v_{1}\left(\lambda k_{\perp}+\kappa_{1}\right) /\left(\epsilon+\Delta_{1}\right)=v_{2}\left(\lambda k_{\perp}-\kappa_{2}\right) /\left(\epsilon+V+\Delta_{2}\right)
$$

Equation (4) can also be written in the form $\kappa_{1} \kappa_{2}=\epsilon_{12}\left(\epsilon, k_{\perp}\right)$, where we have defined $\epsilon_{12}\left(\epsilon, k_{\perp}\right)=\left(\epsilon^{2}+\epsilon V-\Delta_{1} \Delta_{2}\right) /\left(v_{1} v_{2}\right)-k_{\perp}^{2}$. This requires $\epsilon_{12}\left(\epsilon, k_{\perp}\right)>0$, which implies $|\epsilon|>\left|\epsilon_{0}\left(k_{\perp}\right)\right|$, where $\epsilon_{0}\left(k_{\perp}\right)$ solves $\epsilon_{12}\left(\epsilon_{0}, k_{\perp}\right)=0$. From (4) one obtains the IS energy $\epsilon_{\lambda}$ as a function of the momentum $k_{\perp}$,

$$
\epsilon_{\lambda}\left(k_{\perp}\right)=\left[-V\left(k_{\perp}^{2} v_{1} v_{-}+\Delta_{1} \Delta_{-}\right)-\lambda k_{\perp} D\left(k_{\perp}^{2} v_{-}^{2}+\Delta_{-}^{2}-V^{2}\right)^{1 / 2}\right] /\left(k_{\perp}^{2} v_{-}^{2}+\Delta_{-}^{2}\right)
$$

where $v_{-}=v_{1}-v_{2}, \Delta_{-}=\Delta_{1}-\Delta_{2}$, and $D=\Delta_{2} v_{1}-\Delta_{1} v_{2}$. The localized states exist in a region of momenta $\min \left\{k_{\perp i}^{(\lambda)}\right\}<k_{\perp}<\max \left\{k_{\perp i}^{(\lambda)}\right\}$, where the end points 
$k_{\perp i}^{(\lambda)}$ are determined by the intersection of the curves $\epsilon_{0}\left(k_{\perp}\right)$ with the bulk dispersion curves $\epsilon_{1,2}\left(k_{\perp}\right)$, respectively,

$k_{\perp i}^{(\lambda) 2}=\left[V^{2} v_{i}-2 v_{-} \Delta_{i} \Delta_{-}+\operatorname{sgn}\left(\lambda k_{\perp}\right) V\left(4 \Delta_{i} v_{-} D+V^{2} v_{i}^{2}\right)^{1 / 2}\right] /\left(2 v_{i} v_{-}^{2}\right), \quad i=1,2$.

Equation (5) has been obtained without imposing any restrictive conditions on the parameters $v_{i}, \Delta_{i}$, and $V$. If $v_{-}=0$, one recovers from (5) the results of Refs. [3, [4], viz. that IS with linear dispersion exist in the band-inverted case $\left(\Delta_{1} \Delta_{2}<0\right)$. Moreover, relation (6) for the end points reduces to $k_{\perp i}^{(\lambda) 2}=\left(\Delta_{-}^{2}-V^{2}\right) \Delta_{i}^{2} / V^{2} v^{2}$, showing that the IS exist in a finite range of $k_{\perp}$ for nonzero work function offset $V \neq 0$ (cf. 四).

On the other hand, if $v_{-} \neq 0$, Eq. (5) describes a novel type of IS which has a nonlinear dispersion and which, in particular, occurs in junctions without band inversion. For this, it is necessary that a crossing of the bulk dispersion curves occurs. This is seen immediately for an unshifted heterojunction with $V=0$ where (6) has a real solution $k_{\perp i}^{(\lambda)}$ only if $\Delta_{-} v_{-}<0\left(\Delta_{1}<\Delta_{2}, v_{1}>v_{2}\right.$ or $\Delta_{1}>\Delta_{2}$, $\left.v_{1}<v_{2}\right)$, i.e. if the bulk dispersion curves $\epsilon_{i}^{( \pm)}\left(k_{\perp}\right)$ intersect.

The behavior of the IS as a function of the offset $V$ is illustrated in Fig. 1 for the following parameters: $\Delta_{1}=240 \mathrm{meV}, \Delta_{2}=300 \mathrm{meV}, v_{1}=2 \times 10^{8} \mathrm{~cm} \mathrm{~s}^{-1}, v_{2}=10^{8}$ $\mathrm{cm} \mathrm{s}^{-1}$. The IS lie in the vicinity of the crossing point of the bulk dispersions. It is there where their wave functions are well localized ( $\kappa_{i}$ "large"). Moving away from this point the energy of the IS approaches the bulk dispersion curves so that the wave functions become less localized. We see that the IS are shallow: for small values of $V$ the IS energies $\epsilon_{-1}\left(k_{\perp}\right)$ and $\epsilon_{+1}\left(k_{\perp}\right)$ lie near the bulk dispersions. With increasing $V$ the region $k_{\perp 1}^{(-)}<k_{\perp}<k_{\perp 2}^{(-)}$widens whereas the region $k_{\perp 1}^{(+)}<k_{\perp}<k_{\perp 2}^{(+)}$narrows. For $V<\left|\Delta_{-}\right|$, the heterojunction is of type I, with two IS having opposite $\lambda$ for given $k_{\perp}$, which becomes type II for $V>\left|\Delta_{-}\right|$, with only the IS near the valence band remaining.

The analytic results for an abrupt junction remain virtually the same for graded junctions. Using for $v(z)$ and $\Delta(z)$ a simple representation involving a smoothness 
parameter $d$, we find by numerical calculation that the energy of the IS changes by no more than $\pm 0.5 \mathrm{meV}$ as a function of $d$ up to values as large as $d \approx 3 \times 10^{-6} \mathrm{~cm}$ $\left(\kappa_{i} d \approx 1, \kappa_{i} \approx k_{\perp}\right)$, and thus essentially coincides with the energy obtained for the abrupt contact.

Our case is a typical example of weak localization (characterized by the weak dependence of the IS on $d$ for $\kappa_{i}^{-1} \gg d$ ), similar to what is found in both the one-band and two-band approximations [3]. This is seen from an analysis of the graded junction, which also throws some light on the physical meaning of the MC in the abrupt case. Eliminating $g$ from (2) (written for the functions $f, g$ with $\lambda=+1$ ), one arrives at a Klein-Gordon-type equation for $f$. Using for $f$ the ansatz $f=\exp \left[\int \gamma(z) \mathrm{d} z\right]$, one obtains

$$
\gamma^{2}+\left(\epsilon^{2}-\Delta^{2}\right) / v^{2}-k_{\perp}^{2}+\left[Q\left(k_{\perp}+\gamma\right)\right]^{\prime} / Q=0
$$

with $Q=v /(\Delta+\epsilon)$. Away from the interface $(|z| \gtrsim d)$, the last term on the LHS of $(7)$ vanishes, so that there $\gamma \approx \kappa_{1},-\kappa_{2}$. In the interface region $(|z| \lesssim d), Q^{\prime}$ and $\gamma^{\prime}(\approx \kappa / d)$ predominate over the other terms $\left(\approx \kappa^{2}\right)$, so that $\left.Q\left(k_{\perp}+\gamma\right)\right|_{z_{1}}=$ $\left.Q\left(k_{\perp}+\gamma\right)\right|_{z_{2}}$, where $z_{1}<0, z_{2}>0\left(\left|z_{i}\right| \lesssim d\right)$. Replacing $Q$ and $\gamma$ with their bulk values, $Q\left(z_{i}\right) \rightarrow v_{i} /\left(\epsilon+\Delta_{i}\right), \gamma\left(z_{1}\right) \rightarrow \kappa_{1}, \gamma\left(z_{2}\right) \rightarrow-\kappa_{2}$, we recover Eq. (4) (with $V=0$ ). The last term on the LHS of (7) acts like a potential which turns around the sign of the wave vector $\gamma(z)$ as $z$ passes through the interface. In the abrupt case its role is taken over by the MC.

From our analysis, the following physical picture emerges. The free motion in the interface plane with wave number $k_{\perp} \neq 0$ gives rise to binding in the $z$-direction. The energies of the electron-like IS lie above the bulk conduction band edges $\Delta_{1}$ and $\Delta_{2}-V$ (and analogously for the hole-like IS). Thus, the IS are embedded in the continuum of the carriers; nevertheless, they lie in the gap if one compares their energies with those of the two bulk dispersions for the same value of $k_{\perp}$. The IS originate from the intersecting bulk states, they are "pushed" down into the bandgap owing to the negative energy contribution of the (quantized) motion in the 
$z$-direction.

The principal feature of the eigenvalue equation (4) is that it contains $k_{\perp}$ explicitly, so that in a range of values of $k_{\perp}$, the two exponentially decaying left and right solutions can be matched at $z \approx 0$ when both $\kappa_{i}$ are small. This implies a change of sign of the logarithmic derivative. Formulations which do not take this into account cannot describe our IS [12]. The region $k_{\perp 1}^{(\lambda)}<k_{\perp}<k_{\perp 2}^{(\lambda)}$ in which the IS exist could be quite broad for IV-VI semiconductors as $v$ varies here over a wide range. The III-V semiconductors are generally well described within the approach of [12. However, in extreme cases the values of $v$ differ by as much as $0.2 \times 10^{8} \mathrm{~cm}$ $\mathrm{s}^{-1}$ for materials of this group (cf., e.g., Ref. [14], ch. II, Table III), allowing IS in a small range of $k_{\perp}$.

The essential condition for the existence of the IS in heterojunctions without band inversion, viz. the intersection of the bulk dispersion curves, $\Delta_{-} v_{-}<0$, holds for most narrow-band semiconductors [14]. This is easily understood: the larger the bandgap, the weaker the interband interaction, i.e. materials with large $\Delta$ have small $v$.

In summary, we have used the two-band model to derive unique matching conditions for an abrupt heterojunction. If the interband velocity matrix element $v$ is different in the two semiconductors (as in most heterojunctions), localized interface states occur, in particular in systems without band inversion, if the bulk curves of the semiconductors intersect. This is expected to be the normal case. The effect of the $\mathrm{MC}$ is rather weak, leading to IS which lie near the bulk dispersions. However, in spite of this, it should be possible to identify these states experimentally, since owing to their localized nature, their contribution to transport and optical properties is different from that of the bulk states.

The present model, while being not unrealistic for IV-VI semiconductors, is simple and transparent, and should provide an ideal guideline for more detailed theoretical treatments which are expected to lead to a more quantitative exploration of 
the predicted type of state. Moreover, beyond the present context of the envelope function description, our results represent an important and novel contribution to quantum mechanics in general, as they demonstrate the existence of localized interface states in a situation where, without the manifest occurrence of a binding potential, localization is seemingly impossible.

This material is based upon work supported by the U.S. Civilian Research and Development Foundation under Award No. RP1-273. The present work was supported in part by the Russian Foundation for the Fundamental Research (Projects No. 96-02-16701, 97-02-16346, and 97-02-16042). 


\section{References}

[1] Ohno H., Mendez E. E., Brum J. A., Hong J. M., Agulló-Rueda F., Chang L. L. and Esaki L., Phys. Rev. Lett., 64 (1990) 2555.

[2] Tamm I. E., Phys. Z. Sowjetunion, 1 (1932) 733.

[3] Volkov B. A. and Pankratov O. A., Pis'ma Zh. Eksp. Teor. Fiz., 42 (1985) 145 [Sov. Phys. JETP Lett., 42 (1985) 178]; Pankratov O. A., Pakhomov S. V. and Volkov B. A., Solid State Commun., 61 (1987) 93.

[4] Korenman V. and Drew H. D., Phys. Rev. B, 35 (1987) 6446; Agassi D. and Korenman V. ibid., 37 (1988) 10095.

[5] Tikhodeev S. G., Solid State Commun., 78 (1991) 339.

[6] Davison S. G. and Stȩślicka M., Basic theory of surface states (Oxford Univ. Press, Oxford) 1992.

[7] Sokolov I. M., Zh. Eksp. Teor. Fiz., 89 (1985) 556 [Sov. Phys. JETP, 62 (1985) $317]$.

[8] Morrow R. A. and Brownstein K. R., Phys. Rev. B, 30 (1984) 678.

[9] Keldysh L. V., Zh. Eksp. Teor. Fiz., 45 (1963) 364 [Sov. Phys. JETP, 18 (196 4) 253]; Wolff P. A., J. Phys. Chem. Solids, 25 (1964) 1057.

[10] Idlis B. G. and Usmanov M. Sh., Fiz. Tekh. Poluprovodn., 26 (1992) 329 [Sov. Phys. Semiconductors, 26 (1992) 186].

[11] Kolesnikov A. V. and Silin A. P., Pis'ma Zh. Eksp. Teor. Fiz., 61 (1995) 733 [JETP Lett., 61 (1995) 747]; J. Phys.: Condens. Matter, 9 (1997) 10929.

[12] Bastard G., Phys. Rev. B, 24 (1981) 5693; ibid., 25 (1982) 7584; Bastard G., Wave mechanics applied to semiconductor heterostructures (Les Éditions de Physique, Les Ulis) 1996. 
[13] Burt M. G., Semicond. Sci. Technol., 3 (1988) 739; J. Phys.: Condens. Matter, 4(1992) 6651.

[14] Partin D. L. and Hermans J., Growth of narrow bandgap semiconductors, in Handbook of semiconductors, edited by Mahajan S., Vol. 3a, p. 372 (NorthHolland, Amsterdam) 1994. 


\section{Figure captions}

Figure 1. Dispersion $\epsilon_{\lambda}\left(k_{\perp}\right)$ of an abrupt heterojunction for several values of $V$ (heavy curves; for parameters, see text). Dotted curves: bulk dispersions $\epsilon_{1,2}^{( \pm)}\left(k_{\perp}\right)$. The regions (with end points $k_{\perp i}^{(\lambda)}$ ) where localized IS occur are defined by the condition $\left|\epsilon_{\lambda}\left(k_{\perp}\right)\right|>\left|\epsilon_{0}\left(k_{\perp}\right)\right|$, where $\epsilon_{0}\left(k_{\perp}\right)$ is represented by the thin curves. It is understood that the figures are continued symmetrically across $k_{\perp}=0$ with a reversal of sign of $\lambda$. Inset in a): band-edge profile of the heterojunction; the energy gap is shaded. 


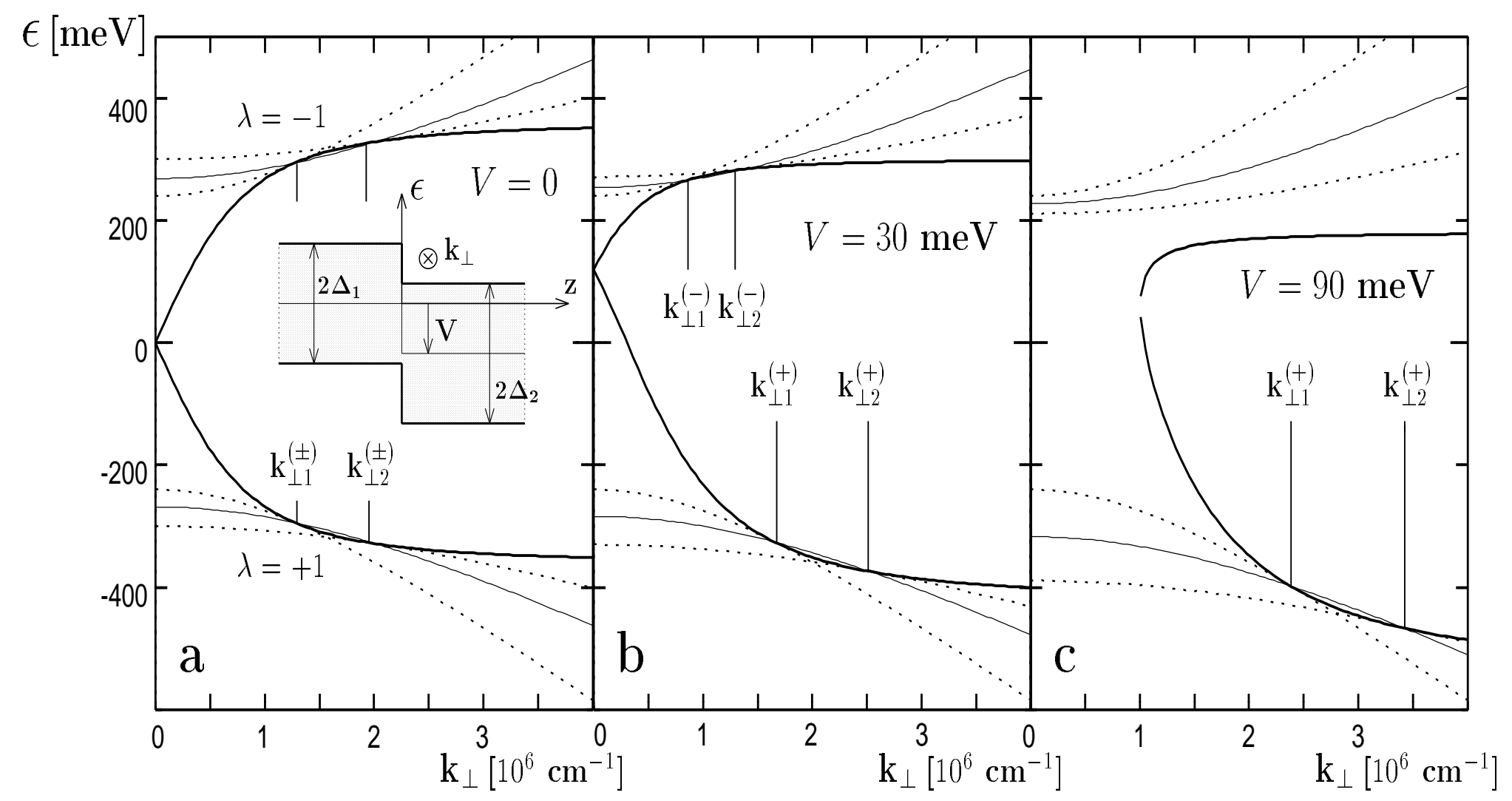

ำ

Figure 1 